\title{
Repetitive transcranial magnetic stimulation significantly influences the eating behavior in depressive patients
}

This article was published in the following Dove Press journal:

Neuropsychiatric Disease and Treatment

\section{Katarína Jaššová \\ Jakub Albrecht \\ Silvie Čerešňáková \\ Hana Papežová \\ Martin Anders}

Department of Psychiatry, First Faculty of Medicine, Charles University and General University Hospital in Prague, Prague 2

121 08, Czech Republic
Correspondence: Katarína Jaššova

Department of Psychiatry, First Faculty of Medicine, Charles University and General

University Hospital in Prague, Ke

Kateřinkám 1394/8, Prague 4149 00,

Czech Republic

Tel +42060856465I

Email katarina.jasso@gmail.com
Background: Depressive syndrome is one of the most common of psychiatric diseases and is ranked as the largest single contributor to global disability. Depression worsens the treatment outcomes of comorbid conditions and is a predictor of an increased mortality rate. Frequent comorbidities accompanying depressive syndrome are eating disorders (ED). The novel brain stimulation technique termed repetitive transcranial magnetic stimulation (rTMS) has been developed as a clinical tool to treat depression. Simultaneously the effect of rTMS has been studied on ED.

Purpose: The aim of this study was to monitor the correlation between the improvement in depressive symptoms and changes in eating behavior after rTMS treatment, and potential possibility of the utilization of rTMS in the treatment of these frequent comorbid conditions. Methods: To map the change in eating behavior, this study follows the changes in answers 5 and 7 in the Zung Self-Rating Depression Scale. The patients were treated with highfrequency rTMS focused on the left dorsolateral prefrontal cortex.

Results: We observed a significant change in both questions. At the same time, the change in both questions correlates with a variance in the overall depressive symptoms.

Conclusion: The rTMS treatment of depressive syndrome resulted in significant clinical improvements, including changes in eating behavior.

Keywords: depressive syndrome, eating disorders, eating behavior, rTMS, Zung Self-Rating Depression Scale

\section{Introduction}

Depressive syndrome is one of the most common of psychiatric diseases, ${ }^{1}$ as well as contributing to significant disabilities and adverse socioeconomic effects. ${ }^{2}$ According to the World Health Organization, depression is ranked as the largest single contributor to global disability (7.5\% of all years lived with disability in 2015$){ }^{1}$

Moreover, comorbidities accompanying depressive syndrome are frequent. ${ }^{3-5}$ Depression worsens the treatment outcomes of these comorbid conditions, ${ }^{6}$ and is an independent predictor of an increased mortality rate. ${ }^{7,8}$ Therefore, there are at least three reasons for studying these comorbidities. The co-occurrence of two or more disorders may have nosological implications, implications for etiologic models, and negative consequences on various aspects of life. ${ }^{9}$

Eating disorders (ED) are frequent comorbidities of the depressive syndrome. ${ }^{10,11}$ For example, the lifetime prevalence of "at least one mood disorder" varies from $24 \%$ to $90 \%$ in patients with bulimia nervosa $(\mathrm{BN}),{ }^{12,13}$ and from $31 \%$ to $88.9 \%$ in patients suffering 
from anorexia nervosa (AN). ${ }^{14,15}$ The chronology of onset of depression and ED remains unclear. ${ }^{16}$ However, the relationship between the illnesses is indisputable, ${ }^{10,16}$ and some studies even suggest a shared genetic pathway between ED and depression (Afari, N. et al, 2010; Mas, S. et al, 2013). ${ }^{17,18}$

In the last 20 years, a novel brain stimulation technique termed repetitive transcranial magnetic stimulation has been developed as a clinical tool to treat depression. ${ }^{19}$ Several previous meta-analyses have demonstrated the antidepressant efficacy of high-frequency rTMS (5-20 Hz) applied to the left dorsolateral prefrontal cortex (DLPFC). ${ }^{20-22}$ Simultaneously the effect of rTMS treatment has been studied on ED, in recent years primarily on AN. ${ }^{23,24}$ The effect on craving in $\mathrm{BN}$ as well as binge eating disorder (BED) has already been established..$^{25,26}$ In the literature, one can even find a case report of women with depression and comorbid $\mathrm{BN}$, for whom rTMS proved to have a positive effect. ${ }^{27}$

The common target of stimulation, in the case of studies following alternative treatment of ED, is the left DLPFC. Generally, DLPFC is involved in decision-making and emotion regulation, ${ }^{28}$ which play roles in the symptomatology of ED. For example, in AN, hypoactivity of prefrontal cortical regions during response inhibition and set-shifting tasks has been reported. ${ }^{29}$ Simultaneously, functional neuroimaging studies of AN patients using symptoms-provocation paradigms have proposed either aberrant functioning of "top-down" prefrontal regions (executive control) and/or subcortical regions promoting "bottom-up" (stimulus-driven) responses, anxiety-related mesolimbic circuits, reward-related regions (eg, the striatum), and parietal somatosensory regions. $^{30}$ Therefore, the increase of the activity of DLPFC may improve anorexic eating behavior. ${ }^{23}$

While studies describing the relationships between BED, $\mathrm{BN}$, obesity, and depression are common, ${ }^{10-14,16-18,31,32}$ studies discussing changes of appetite during depression are rare, despite the fact that a reduction in food intake and weight loss are important physical symptoms of depression, paradoxically currently primarily associated frequently with depressed women. $^{33}$
According to our knowledge, there are no studies which specifically evaluate the changes in eating habits in depressive patients after rTMS treatment. This study follows the changes in answers 5 and 7 in the Zung SelfRating Depression Scale (ZDS), to map the subjective perception of eating behavior and weight change.

The Geliebter study (2001), which refers the change in meal intake in obese binge eaters in relation to mood and gender, eliminated question 5 (I eat as much as I used to) and question 7 (I notice that I am losing weight) from the ZDS because they address decreased eating and weight loss associated with depression, which was not applicable to their study's purpose. ${ }^{34}$ By contrast, for our study's aim, we used exactly these two questions to follow-up the changes in eating habits, as represented by appetite and body weight. Furthermore, we also have followed the interrelationship of these two aspects eating, and their association to changes of SDS Index, the duration of the stimulation, and the relationship with the diagnoses.

\section{Materials and methods}

The study was conceived as observational, pragmatic, open, non-randomized clinical evaluation. Research of rTMS application in depressive patients had been approved by Ethical Committee of General University Hospital in Prague with No.f1933/18 S-IV.

\section{Participants}

The participants in our study were both outpatients and inpatients being treated for depressive syndrome at the Department of Psychiatry First Faculty of Medicine Charles University and General University Hospital in Prague, Czech Republic (Table 1). The inclusion criteria for rTMS treatment were depressive syndrome of large scale of affective disorders: Bipolar Affective Disorder (12.5\%), Depressive episode (44.54\%), Organic Depressive Disorder (8.93\%), Recurrent Depressive Disorder (14.29\%), and Mixed anxiety and depressive disorder (19.64\%) (Table 1, Figure 1).

Table I Demographic and clinical characteristics

\begin{tabular}{|l|l|l|l|l|l|l|l|l|l|}
\hline \multirow{2}{*}{ Participants } & \multicolumn{4}{l}{ Gender } & \multicolumn{3}{l|}{ Type of hospitalization } & \multicolumn{3}{l|}{ Diagnoses } \\
\cline { 2 - 10 } & Men & Women & Inpatients & Outpatients & BAD & DE & OD & RD & ADD \\
\hline N & 31 & 25 & 26 & 30 & 7 & 25 & 5 & 8 & 11 \\
$\%$ & 55.36 & 44.64 & 46.43 & 53.57 & 12.5 & 44.64 & 8.93 & 14.29 & 19.64 \\
\hline
\end{tabular}

Abbreviations: BAP, Bipolar Affective Disorder; DE, Depressive episode; OD, Organic Depressive Disorder; RD, Recurrent Depresve Disorder, ADD, Mixed anxiety and depressive disorder. 


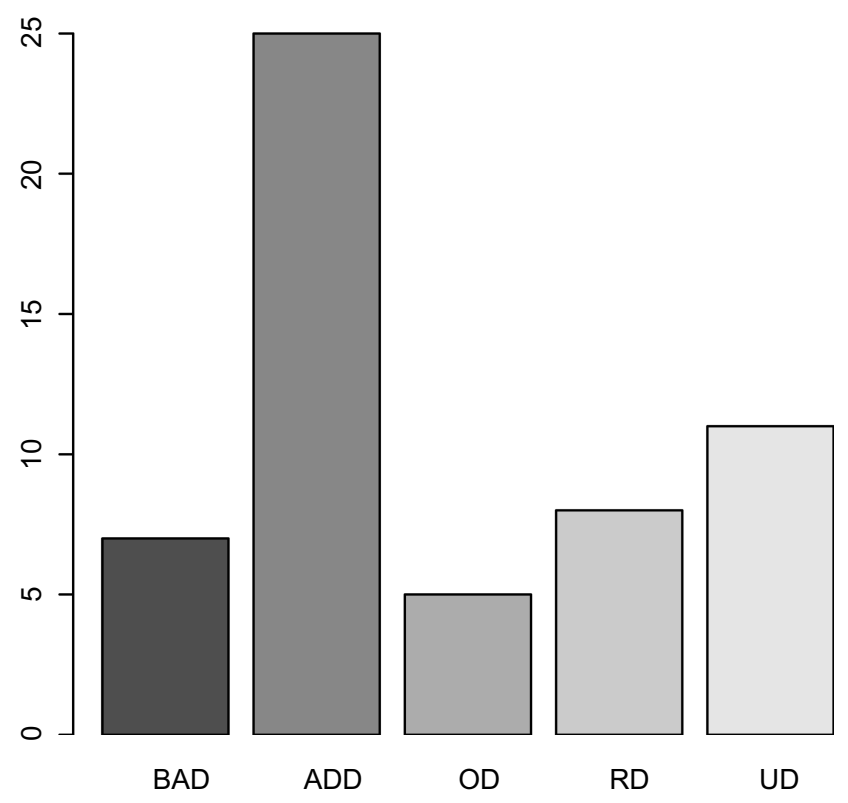

Figure I The scale of affective disorders in the file.

Abbreviations: BAD, bipolar affective disorder; DE, depressive episode; OD, organic depressive disorder; RD, recurrent depressive disorder; ADD, mixed anxiety and depressive disorder.

Table 2 Medication

\begin{tabular}{|l|l|l|}
\hline \multirow{2}{*}{ Medications } & Participants \\
\cline { 2 - 3 } & N & $\%$ \\
\hline bzd & 31 & 57.41 \\
hyp & 6 & 11.11 \\
Li & 5 & 9.26 \\
API & 9 & 16.67 \\
AP2 & 26 & 48.15 \\
SARI & 6 & 11.11 \\
SSRI & 21 & 38.89 \\
SNRI & 19 & 35.19 \\
TeCA & 14 & 25.93 \\
vort & 1 & 1.85 \\
antiH & 4 & 7.41 \\
M & 5 & 9.26 \\
RIMA & I & 1.85 \\
IChAE & I & 1.85 \\
noo & 1 & 1.85 \\
bup & 4 & 7.41 \\
5HTIA & 3 & 5.56 \\
biper & 1 & 1.85 \\
TCA & 2 & 3.7 \\
\hline
\end{tabular}

Abbreviations: bzd, benzodiazepines; hyp, hypnotics; Li, Lithium; API, Ist Generation of antipsychotics; AP2, second generation of antipsychotics; SARI, serotonin antagonists and reuptake inhibitors; SSRI, selective serotonin reuptake inhibitors; SNRI, serotonin-norepinephrine reuptake inhibitors; TeCA, tetracyclic antidepressants; vort, vortioxetin; antiH, antihistamines; M, melatonin; RIMA, reversible inhibitors of monoamine oxidase; IChAE, cholinesterase inhibitors; noo, nootropics; $5 \mathrm{HTIA}$, antagonists of $5 \mathrm{HTI}$ receptor; biper, biperiden hydrochlorid; TCA, tricyclic antidepressants; bup, bupropion.
The medications of participants varied based upon their diagnoses from antidepressants to antipsychotics or thymostabilizers (Table 2). However, the subjects continued using their habitual medication during the rTMS treatment unchanged to eliminate its clinical effect on overall mental status and thus the results of the study.

The only exclusion criterion was the contraindication to rTMS as history of epileptic paroxysmus, increased intracranial pressure or brain tumor, implanted metal devices in cranium(except oral cavity) and implanted pacemaker or drug pump.

All of the patients had signed the informed consent form as a standard procedure before the start of the treatment by rTMS.

\section{Evaluation of psychopathology changes}

To evaluate the severity of depression, Zung's Self-Rating Depression Scale, ${ }^{35}$ consisting of 20 questions, was used, including emotional, behavioral, cognitive, and somatic depressive symptoms. The subjects rated each item according to how frequently they had felt each of these during the proceeding week. Rating was (1) for none of the time or infrequently, up to (4) for most or all of the time. Summing the scores for all of the individual items gave a total score ranging from 20 to 80 . The raw score converted to a 100-point scale is the SDS/SD Index. The classifications according to the final SDS Index are as follows: those below 49 are considered normal, those between 50 and 59 are considered to have mild depression, 60-69 to have moderate or marked depression, and those who score 70 and above are considered to have severe depression. ${ }^{35-39}$ All subjects completed the questionnaires twice, before and after stimulation.

To evaluate the change in the eating behavior, questions 5 and 7 from ZDS were used.

\section{Stimulation methods}

The average duration of the stimulation was 12.74 sessions (minimum 9 sessions and maximum 24 sessions). There was one non-standard long-term stimulation (24 sessions) in the study. We continued with this stimulation to achieve remission of the depressive symptoms, in accord with the desires of the patient. This is reflected in the results.

The patients were treated with high-frequency rTMS $(10 \mathrm{~Hz})$. Stimulations were realized five times a week, excepting weekends. The other parameters of stimulation were 
determined with snf 15 trains of $10 \mathrm{~s}$ of 100 pulses with individual intertrain 50-70 s providing a total of 1500 . The intensity of stimulation was set up as individual minimal intensity to induce the response of musculus abductor pollicis brevis, which means $100 \%$ of the minimal motor threshold. The target of the stimulation was the left DLPFC which was localized as $5 \mathrm{~cm}$ anterior to and in the same parasagittal plane (coplanar transposition) as the site of maximal abductor pollicis brevis stimulation. ${ }^{19-22,40-42}$ The localization and frequency of stimulation were analogous to other studies on the treatment of ED by rTMS. ${ }^{23-27}$

\section{Statistical analysis}

Linear models with mixed effects were used to analyze the treatment. In a detailed view of the individual questions, the correction of the achieved levels of tests was carried out using the Holm method. For the analysis of food intake details, the non-parametric statistical methods Fisher's exact test, the Wilcoxon test, and the Spearman correlation coefficient were used. The analysis was calculated using the statistical package R, version 3.2.2, R Core Team (2015). Test levels of less than $5 \%$ were considered statistically significant. $^{43}$

\section{Results}

The final sample consisted of 56 cases, including 51 different participants (5 participants completed 2 stimulation series). Excluding one unusually long stimulation (24 sessions), we obtained sample consisting of 55 cases $(\mathrm{N})$. The average age of participants was 53 years old (range 26-85 years).

The severity of depressive symptoms varied from 44 to 94 points on the SD score (Table 3). The average SDS Index before stimulation was 72 points (med. $=72$ ). After the end of stimulation, the SDS Index changed by median delta $=9$ (Figure 2, Table 3). The average duration of stimulation was 12.7 sessions.

There was a statistically significant reduction of symptoms referred in both, question $5(p=0.008)$ and question 7 $(p=0.021$ ) (Figure 3$)$. The questions are related to the statistically significant level ( $p=0.009$, Table 4$)$. The change in both questions correlates with a variance in the delta SDS Index ( $p=0.046, p=0.00$ ) (Figure 4). The contribution of changes in questions 5 and 7 to overall changes in the SDS Index was unimportant (median 0 and 0.02). None of the questions examined showed significant changes when considering the different diagnoses. If we take into account the unusually long stimulation, and then recalculate the results

Table 3 SDS Index before and after stimulation

\begin{tabular}{|l|l|l|l|l|l|l|l|l|l|}
\hline SDS index & Min. & Ist Qu. & Median & Mean & 3rd Qu. & Max. & SD & N & NA's \\
\hline Before stimulation & 44.00 & 65.0 & 72.0 & 71.43 & 79.0 & 94.0 & II-Oct & 54.00 & 2.0 \\
After stimulation & 34.00 & 51.00 & 59.00 & 60.70 & 68.00 & 88.00 & Dec-39 & 54.00 & 2.0 \\
\hline
\end{tabular}

A

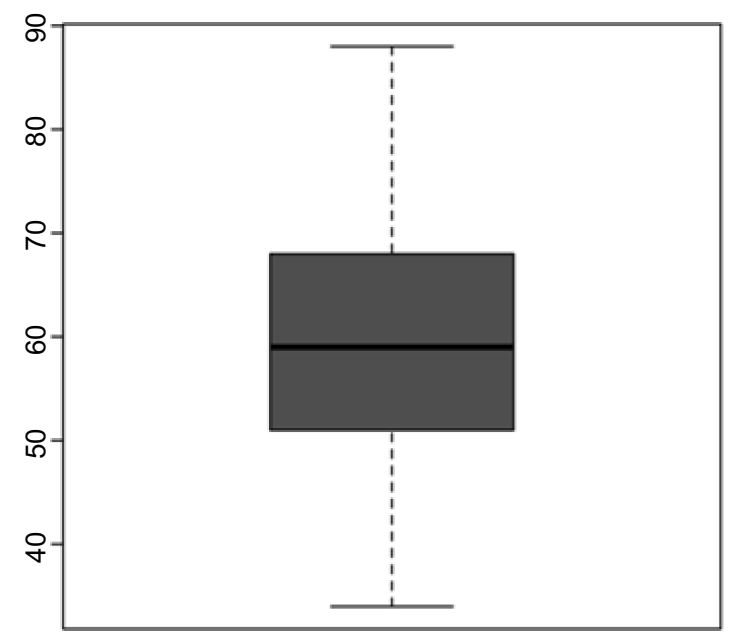

B

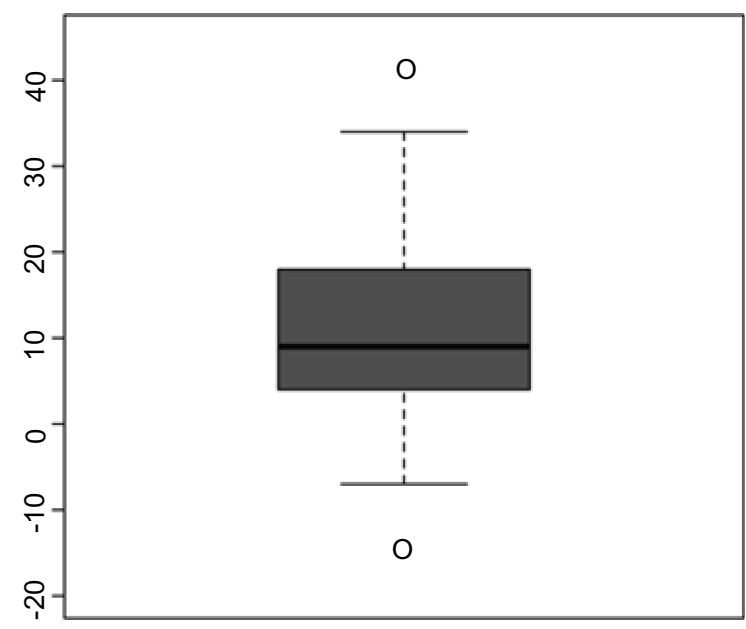

Figure 2 (A) Overall SDS Index after stimulation. (B) Delta SDS Index after stimulation. 


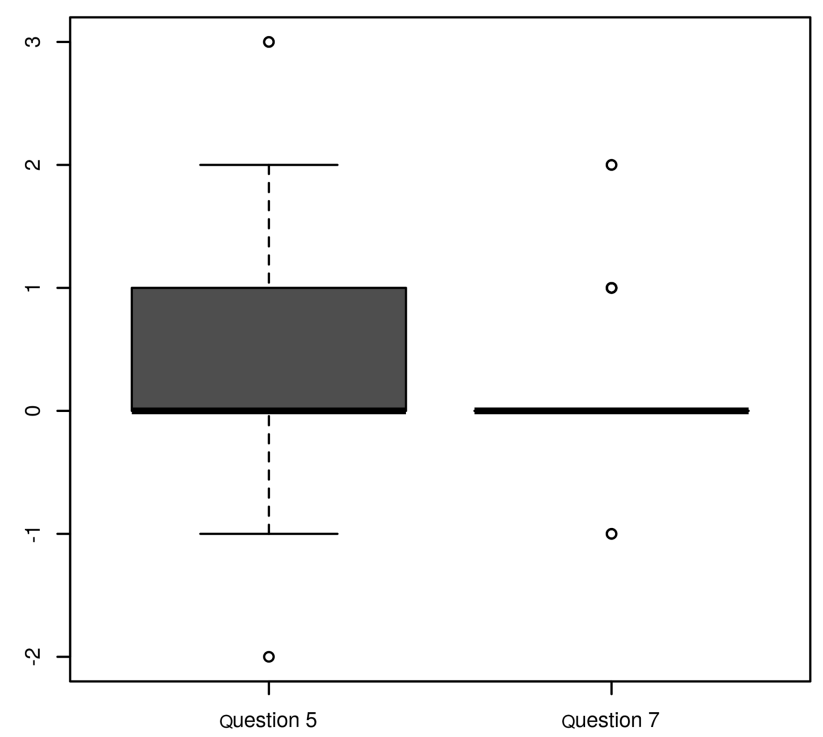

Figure 3 The change in questions 5 and 7 for the file without unusually long stimulation.

Table 4 Correlation between the change in question 5 and question 7 for the file without unusually long stimulation: The test of correlation: $p=0.009$

\begin{tabular}{|l|l|l|l|l|}
\hline-2 & -1 & 0 & 1 & 2 \\
-1 & 0 & 0 & 0 & 1 \\
0 & 0 & 6 & 1 & 0 \\
1 & 2 & 15 & 1 & 0 \\
2 & 0 & 12 & 4 & 0 \\
3 & 0 & 3 & 0 & 1 \\
\hline
\end{tabular}

A

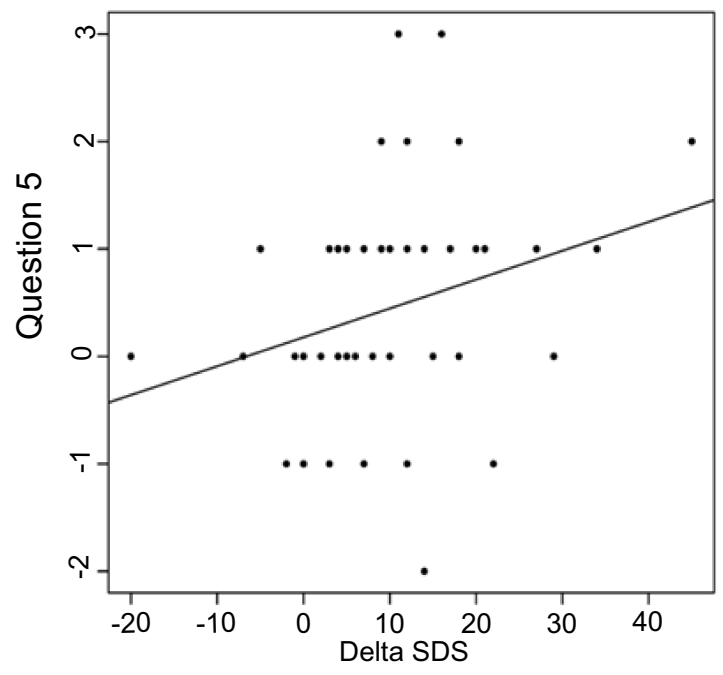

for this sample, the only statistically interesting modifications compared to the previous calculation, emerged in an insignificant change in question number $7(p=0.122)$ (Figure 5).

\section{Discussion}

The relationship between depression and ED is well known, ${ }^{11-18}$ as is the connection between improvements of depressive symptoms and subsequent positive changes in eating behavior. This linkage is more-or-less successfully integrated into the treatment of comorbid ED and depression, for example by using selective serotonin reuptake inhibitors in pharmacotherapy. ${ }^{44}$ Therefore, our aim was not to examine the association between the improvements of depressive symptoms and the eating behavior itself, but rather to document the range of these changes in appetite and body weight after rTMS treatment in depressive patients.

This study's findings confirmed our clinical experiences and assumptions that any improvement of depression and a positive shift in questions 5 and 7 are associated. Considering the wording of the questions, we concluded that the participants improved their eating conditions, specifically the amount of food intake and stabilization of their body weight. Surprisingly, the significance of the positive change in question 7 (concerning body weight) after completion of rTMS treatment emerged by exclusion of the unusually long stimulation from the sample. Based on ongoing studies, where the standard length

B

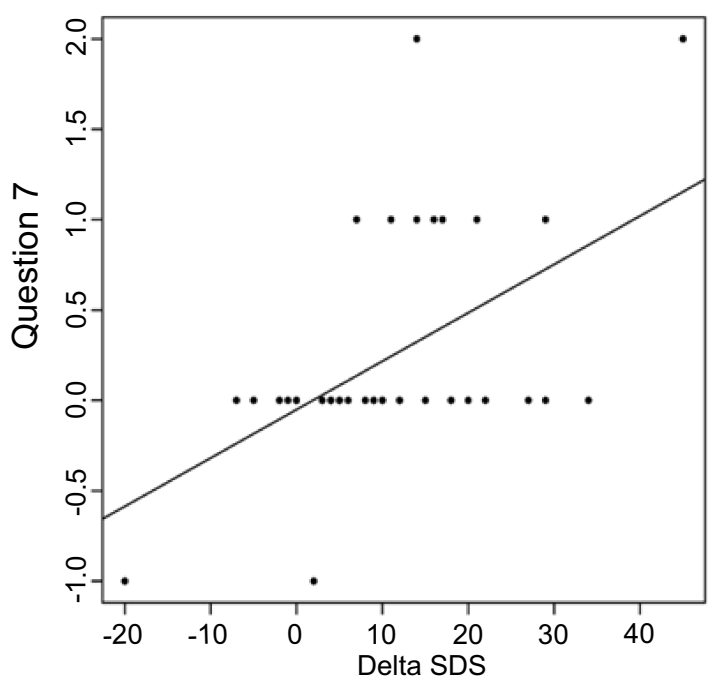

Figure 4 (A) Correlation between the change in question 5 and delta SDS Index for the file without unusually long stimulation. (B) Correlation between the change in question 7 and delta SDS Index for the file without unusually long stimulation. 


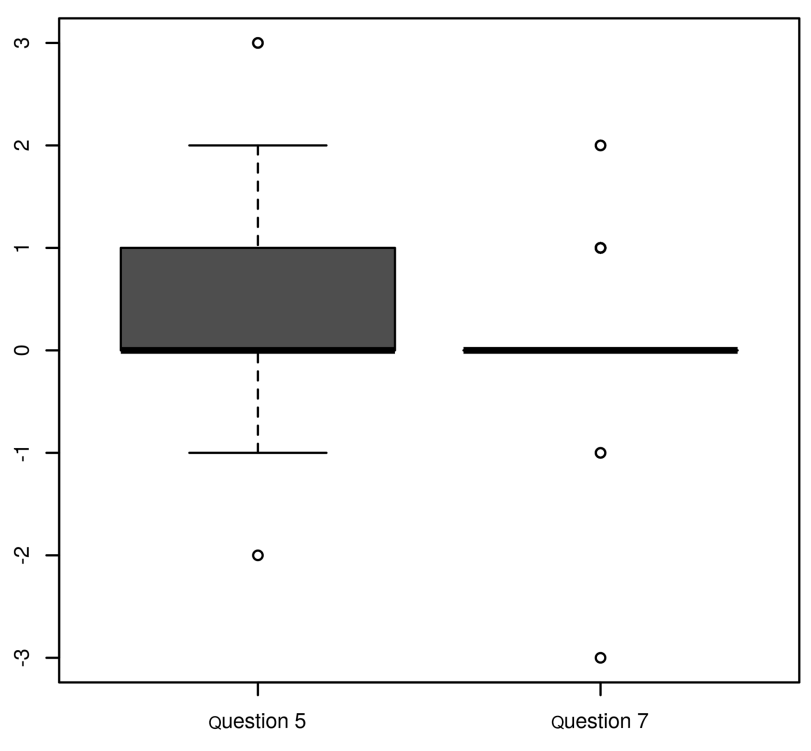

Figure 5 Change in questions 5 and 7 in file with unusually long stimulation.

of stimulation is 20 sessions, ${ }^{23,24}$ we had expected that longer stimulation would have had a better effect, but our results suggest an opposite tendency. The question of any positive effect of longer stimulation still remains open, and should be replicated in further research.

Based on the results of our study, therapy using rTMS can positively influence the course of a wide spectrum of depression, improving depressive symptoms as well as related behavioral factors (reflecting changes in appetite and weight). However, their contribution (questions 5 and 7) in the overall changes in SDS Index was negligible. Thus, a positive result upon depression improves eating patterns and not vice versa.

Additionally, we examined if the change in overall SDS Index and the eating behavior questions differed between the affective diagnoses included in the study, as defined by International Classification of Diseases, 10th Revision (ICD-10), however we did not find any statistically significant difference. Therefore, we hypothesize, that a positive change in eating behavior after rTMS treatment could be expected upon the entire spectrum of depressive diagnoses.

In 2013, Jianjun Chen compared the efficacy of highfrequency left-DLPFC rTMS with low-frequency rightDLPFC rTMS treatment of depression in a meta-analysis of seven randomized controlled trial studies with 221 adult subjects. No significant differences between the examined treatments were found. ${ }^{44}$ Also, the small meta-analysis performed by Enokibara et al, recently proved that highfrequency right-DLPFC rTMS is more successful in the relief of craving in substance addictions ${ }^{45}$ which are neurobiologically related to ED. ${ }^{46}$ In our opinion, it would be interesting to examine the effect of rTMS on right-DLPFC on the eating behavior in depressive patients, and its possible use in the treatment of dual diagnoses.

There are some limitations of this study. First of all, the number of participants is relatively small. Secondly, the study was conceived as observational, pragmatic, open, non-randomized clinical evaluation. A larger, placebo controlled, and randomized study is necessary to confirm our findings. We were observing the change in specific two questions from ZDS because of their connection with eating disorder. The study would be improved if two different questionnaires would be used to evaluate the change in the mood and the change in the eating habits. Specific questionnaire, which would evaluate the changes in the eating behavior could bring more specific results (eg, EDE-Q - Eating Disorder Examination Questionnaire). Further, it would be very interesting to follow-up improvements in appetite and body weight (or other physical conditions), differentiating between specific depressive diagnoses. Another task for enlarged samples could be to compare the effects of different numbers of stimulation, and to set up the optimal number of sessions.

We followed the study of Geliebter, ${ }^{34}$ in which they excluded questions 5 and 7, because weight loss could be an intended result of overweight women. In our study, it would be interesting to find out if there are any differences in outcomes between overweight participants and participants of normal weight. Also, any gender or age differences in treatment responses remain to be answered by further research.

Finally, we have used the ZDS, which is a subjective scale. In our opinion, it is necessary to use some objective evaluation in subsequent studies (such as following changes of daily menu, body weight, anamorphic ...).

\section{Conclusion}

The rTMS treatment of depressive syndrome across ICD-10 diagnoses of depressive disorder resulted in significant clinical improvements measured by the Zung Self-Rating Scale, including changes in those questions (5 and 7) reflecting eating behavior. However, the changes in eating behavior symptoms did not significantly contribute to the overall improvement of depression. Our findings regarding the characteristics of appetite and weight responses in the treatment of large-scale depressive symptoms bring some new evidence for further studies of rTMS treatment of comorbid ED and depression. 


\section{Acknowledgment}

This work was supported by: GA UK 173915, NPU LO1611, AZV 17-28905A, Progres Q27, MZ ČR - RVO VFN64165.

\section{Disclosure}

Dr. Katarína Jaššová report grants from Charles University in Prague, grants from Ministry of Education Youth and Sports of the Czech Republic, grants from Ministry of Health the Czech Republic, during the conduct of the study. The authors report no other conflicts of interest in this work.

\section{References}

1. World Health Organization. Depression and other common mental disorders. 2017. Available from: http://apps.who.int/iris/bitstream/10665/ 254610/1/WHO-MSD-MER-2017.2-eng.pdf?ua=1/. Accessed June 26, 2018.

2. Kupfer DJ, Frank E, Phillips ML. Major depressive disorder: new clinical, neurobiological, and treatment perspectives. Lancet 2012;379:1045-1055. doi:10.1016/S0140-6736(11)60602-8

3. Kupfer DJ, Frank E. Comorbidity in depression. Acta Psychiatr Scand. 2003;108(418):57-60. doi:10.1034/j.1600-0447.108.s418.12.x

4. Merikangas KR, Kalaydjian A. Magnitude and impact of comorbidity of mental disorders from epidemiologic surveys. Curr Opin Psychiatry. 2007;20(4):353-358. doi:10.1097/YCO.0b013e3281c61dc5

5. Smith DJ, Court H, McLean G, et al. Depression and multimorbidity: cross-sectional study of $1,751,841$ patients in primary care. $J$ Clin Psychiatry. 2014;75(11):1202-1208. doi:10.4088/JCP.14m09147

6. Moussavi S, Chatterji S, Verdes E, Tandon A, Patel V, Ustun B. Depression, chronic diseases, and decrements in health: results from the World Health Surveys. Lancet. 2007;370(9590):851-858 doi:10.1016/S0140-6736(07)61415-9

7. Cuijpers P, Smit F. Excess mortality in depression: a meta-analysis of community studies. J Affect Disord. 2002;72(3):227-236. doi:10.1016/ S0165-0327(01)00413-X

8. Hofmann M, Köhler B, Leichsenring F, Kruse J. Depression as a risk factor for mortality in individuals with diabetes: a meta-analysis of prospective studies. PLoS One. 2013;8(11):e79809. doi:10.1371/journal.pone.0079809. PMID: 24278183.

9. Essau CA. Comorbidity of anxiety disorders in adolescents. Depress Anxiety. 2003;18:1-6. doi:10.1002/da10107

10. Casper RC. Depression and eating disorders. Depress Anxiety. 1998;8 Suppl 1(1):96-104.

11. Ulfvebrand S, Birgegard A, Norring C, Högdahl L, von HausswolffJuhlin Y. Psychiatric comorbidity in women and men with eating disorders results from a large clinical database. Psych Res. 2015;230:294-299. doi:10.1016/j.psychres.2015.09.008

12. Hatsukami D, Eckert E, Mitchell JE, Pyle R. Affective disorder and substance abuse in women with bulimia. Psychol Med. 1984;14(3):701704.

13. Hudson JI, Pope HG, Yurgelun-Todd D. Bulimia and major affective disorder: experience with 105 patients. Psychiatry Psychobiol. 1988;3:37-47.

14. Laessle RG, Kittl S, Fichter MM, Wittchen HU, Pirke KM. Major affective disorder in anorexia nervosa and bulimia. A descriptive diagnostic study. Br J Psychiatry. 1987;151:785-789. doi:10.1192/ bjp. 151.6 .785

15. Fornari V, Kaplan M, Sandberg DE, Matthews M, Skolnick N, Katz JL. Depressive and anxiety disorders in anorexia nervosa and bulimia nervosa Int J Eat Disord. 1992;12:21-29. doi:10.1002/(ISSN)1098-108X
16. Godart N, Radon L, Curt F, Duclos J, Perdereau F, Lang F. Mood disorders in eating disorder patients: prevalence and chronology of ONSET. $J$ Affect Disord. 2015;185:115-122. doi:10.1016/j.jad.2015.06.039

17. Afari N, Noonan C, Goldberg J, et al. Depression and obesity: do shared genes explain the relationship? Depress Anxiety. 2010;27:799806. doi:10.1002/da. 20704

18. Mas S, Plana MT, Castro-Fornieles J, et al. Common genetic background in anorexia nervosa and obsessive compulsive disorder: preliminary results from an association study. $J$ Psychiatr Res. 2013;47 (6):747-754. doi:10.1016/j.jpsychires.2012.12.015

19. Brunoni AR, Fregni F. Clinical trial design in non-invasive brain stimulation psychiatric research. Int $J$ Methods Psychiatric Res. 2011;20(2):19-30. doi:10.1002/mpr.338

20. Dell'osso B, Camuri G, Castellano F, et al. Meta-review of metanalytic studies with repetitive transcranial magnetic stimulation (rTMS) for the treatment of major depression. Clin Pract Epidemiol Ment Health. 2011;7:167-177. doi:10.2174/17450179 01107010167

21. Kozel FA, George MS. Meta-analysis of left prefrontal repetitive transcranial magnetic stimulation. $J$ Psychiatr Pract. 2002;8 (5):270-275

22. Schutter DJ. Antidepressant efficacy of high-frequency transcranial magnetic stimulation over the left dorsolateral prefrontal cortex in double-blind sham-controlled designs: a meta-analysis. Psychol Med. 2009;39(1):65-75. doi:10.1017/S0033291708003462

23. McClelland J, Kekic M, Bozhilova N, et al. A randomised controlled trial of neuronavigated repetitive transcranial magnetic stimulation in anorexia nervosa. PLoS One. 2016:1-17. doi:10.1371/journal. pone. 0148606

24. Van den Eynde F, Guillaume S, Broadbent H, Campbell IC, Schmidt U. Repetitive transcranial magnetic stimulation in anorexia nervosa: a pilot study. Eur Psychiatry. 2013;28(2):98-101. doi:10.1016/j. eurpsy.2011.06.002

25. Uher R, Yoganathan D, Mogg A, et al. Effect of left prefrontal repetitive transcranial magnetic stimulation on food craving. Biol Psychiatry. 2005;58(10):840-842. doi:10.1016/j.biopsych.2005.05.043

26. Van Den Eynde F, Claudino AM, Mogg A, et al. Repetitive transcranial magnetic stimulation reduces cue-induced food craving in bulimic disorders. Biol Psychiatry. 2010;67(8):793-795. doi:10.1016/j. biopsych.2009.11.023

27. Hausmann A, Mangweth B, Walpoth $M$, et al. Repetitive transcranial magnetic stimulation (rTMS) in the double-blind treatment of a depressed patient suffering from bulimia nervosa: a case report. Int $J$ Neuropsychopharmacol. 2004;7:371-373. doi: $10.1017 / \mathrm{S} 1464445704004420$

28. Ochsner KN, Gross JJ. The neural architecture of emotion regulation. Handb Emotion Regul. 2007;1(1):7-109.

29. Sato Y, Saito N, Utsumi A, et al. Neural basis of impaired cognitive flexibility ina patients with anorexia nervosa. PLoS One. 2013;8(5): e61108. doi:10.1371/journal.pone.0061108

30. Brooks SJ, Owen G, Uher R, et al. Differential neural responses to food images in women with bulimia versus anorexia nervosa. PLoS One. 2011;6(7):e22259. doi:10.1371/journal.pone.0022259

31. Huang C, Momma H, Cui Y, et al. Independent and combined relationship of habitual unhealthy eating behaviors with depressive symptoms: a prospective study. $J$ Epidemiol. 2017;27:42-74. doi:10.1016/j.je.2016.08.005

32. Toker S, Shirom A, Melamed S. Depression and the metabolic syndrome, gender-dependent associations. Depress Anxiety. 2008;25:661-669. doi:10.1002/da.20379

33. American Psychiatric Association Diagnostic and Statistical Manual of Mental Disorders. 5th ed. Arlington (VA): American Psychiatric Publishing; 2013.

34. Geliebter A, Hassid G, Hashim SA. Test meal intake in obese binge eaters in relation to mood and gender. Int $J$ Eat Disord. 2001;29:488-494. 
35. Zung WW. A self-rating depression scale. Arch Gen Psychiatry. 1965;12:63-70. doi:10.1001/archpsyc.1965.01720310065008

36. Biggs JT, Wylie LT, Ziegler VE. Validity of the Zung Self-rating Depression Scale. Br J Psychiatry. 1978;132:381-385. doi:10.1192/ bjp.132.4.381

37. Colón de Martí LN, Guzmán Yunqué FS, Guevara-Ramos LM. Early detection of depression using the Zung Self-rating Depression Scale. P R Health Sci J. 1997;16:375-379.

38. Master RS, Zung WW. Depressive symptoms in patients and normal subjects in India. Arch Gen Psychiatry. 1977;34:972-974. doi:10.1001/archpsyc.1977.01770200110016

39. Moschos M, Nitoda E, Lavaris A. Estimation of depression prevalence in patients with Stargardt disease using PHQ-9 and Zung scores. Eur J Ophthalmol. 2016;26(3):268-272. doi:10.5301/ejo.5000700

40. Albrecht J, Mareš T, Jaššová K, Raboch J, Anders M. Stimulation parameters of repetitive transcranial magnetic stimulation in the treatment of neuropsychiatric disorders - protocol and location. Ces Slov Psych. 2017;113(4):158-165.

41. George MS, Lisanby SH, Avery D, et al. Daily left prefrontal transcranial magnetic stimulation therapy for major depressive disorder: a sham-controlled randomized trial. Arch Gen Psychiatry. 2010;67 (5):507-516. doi:10.1001/archgenpsychiatry.2010.46
42. Padberg F, Zwanzger P, Thoma H, et al. Repetitive transcranial magnetic stimulation (rTMS) in pharmacotherapy-refractory major depression: comparative study of fast, slow and sham rTMS. Psychiatry Res. 1999;88(3):163-171.

43. R Core Team. R: A Language and Environment for Statistical Computing. Vienna (Austria): $\mathrm{R}$ Foundation for Statistical Computing; 2015. Available from: www.R-project.org. Accessed August 7, 2019.

44. Chen J, Zhou C, Wu B, et al. Left versus right repetitive transcranial magnetic stimulation in treating major depression: a meta-analysis of randomised controlled trials. Psych Res. 2013;210(3):1260-1264. doi:10.1016/j.psychres.2013.09.007

45. Enokibara M, Trevizol A, Shiozawa P, Cordeiro Q. Establishing an effective TMS protocol for craving in substance addiction: is it possible? Am J Addict. 2016;25:28-30. doi:10.1111/ajad.12 309

46. Hadad NA, Knackstedt LA. Addicted to palatable foods: comparing the neurobiology of Bulimia Nervosa to that of drug addiction. Psychopharmacology. 2014;231:1897-1912. doi:10.1007/s00213014-3461-1

\section{Publish your work in this journal}

Neuropsychiatric Disease and Treatment is an international, peerreviewed journal of clinical therapeutics and pharmacology focusing on concise rapid reporting of clinical or pre-clinical studies on a range of neuropsychiatric and neurological disorders. This journal is indexed on PubMed Central, the 'PsycINFO' database and CAS, and is the official journal of The International Neuropsychiatric Association (INA). The manuscript management system is completely online and includes a very quick and fair peer-review system, which is all easy to use. Visit http://www.dovepress.com/testimonials.php to read real quotes from published authors. 